\title{
The identification of urinary biomarkers for malaria in mouse model
}

\begin{abstract}
Malaria is one of the most prevalent infectious diseases in tropical and subtropical regions. Effective biomarkers are urgently needed. Biomarker is measurable changes associated with physiological and/or pathophysiological conditions. Urine can be better source for biomarker discovery because it accumulates the changes of body while body removes them from blood by homeostatic mechanisms. In this study, we attempted to hunt for urinary biomarkers for malaria using mice infected with Plasmodium yoelii. The urine of mice was collected before infection and 4, 5 days after infection, separated by SDS-PAGE, digested in-gel based on molecular weight and identified using nanoLC-MS/MS. Unexpectedly, although more than $10 \%$ of erythrocytes were infected by $P$. yoelii 5 days after infection, there is no reliable parasite protein were identified even at low MW range, indicating that extrinsic protein may be degraded in blood. In the urine of 4 (about $3 \%$ P. yoelii-infected erythrocytes) and 5 days after infection, 427 and 821 mouse proteins were identified, respectively; 21 and 83 proteins were significantly altered after quantified by Scaffold under the criteria $p<0.05$, fold change $>2$ and the spectral count $>5$. There are only 4 differential proteins shared on both $4^{\text {th }}$ and $5^{\text {th }}$ days after infection and all of them are significantly increased after infection. On the $4^{\text {th }}$ after infection, there is no obvious renal damage; however, on the $5^{\text {th }}, 26$ of the 83 proteins (especially 20 of the 40 high abundant proteins) are biomarkers for renal diseases, indicating that the kidneys are seriously injured. All these 4 proteins (Mucin-2, Galectin-3-binding protein, Ig alpha chain C region, Apolipoprotein D) participate in the defense against pathogens and the regulation of tissue inflammation In conclusion, although there are no parasite proteins identified in the urine, the host (mice) urine proteins may be candidate biomarkers for malaria.
\end{abstract}

Keywords: LC-MS/MS, acetonitrile, iodoacetamide, biomarker, pathophysiological process, Plasmodium yoelii
Volume 7 Issue 2 - 2018

\author{
Xundou Li, 'Weiwei Deng,' Youhe Gao, ${ }^{1,2}$ \\ Heng Wang' \\ 'Institute of Basic Medical Sciences, Chinese Academy of Medical \\ Sciences, China \\ ${ }^{2}$ Department of Biochemistry and Molecular Biology, Beijing \\ Normal University, China
}

Correspondence: Youhe Gao, Department of Biochemistry and Molecular Biology, Beijing Normal University, China

No. 19, Xinjiekouwai Street, Beijing, China, Tel 861058804382 Email gaoyouhe@bnu.edu.cn

Received: March 21, 2018 | Published: March 26, 2018

\section{Abbreviations: LC-MS/MS, liquid chromatography-tandem mass; 1D-SDS PAGE, one dimensional sodium dodecyl sulfate polyacrylamide gel electrophoresis; DTT, dithiothreitol; ACN, acetonitrile; IAA, iodoacetamide}

\section{Introduction}

Biomarkers is a measurable changes associated with a physiologica and/or pathophysiological process. In order to maintain normal life activities, the blood is homeostatic and draining into urine is important way of keeping blood stable. Without homeostasis, urine is more likely to reflect changes in the body. In addition, urine can accumulate a variety of changes of the body, making it an ideal biological source for disease biomarker discovery. ${ }^{1,2}$ As reviewed in a recent paper, kinds of physiological changes can be reflected in urine, so it is expect that urine can be used to detect the changes in pathological and/or pharmacological process. ${ }^{3}$ Actually, urine can be more sensitive to physiological changes than blood, ${ }^{4}$ and some biomarkers behave even better in urine than in blood. ${ }^{5-7}$ Many urinary biomarkers have recently been identified for various types of diseases. ${ }^{8}$

However, urinary biomarker research remains challenging because changes in urine are very complicated and the urine is affected by a variety of factors such as gender, ${ }^{9}$ age, medications,,${ }^{10,11}$ exercis, ${ }^{12}$ and smoking ${ }^{13}$ in addition to many other physiological variables and environmental factors. To circumvent these issues, it is important to employ more simple and controllable systems, such as animal models. The animal models can allow for controlling genetic and environmental factors, so it is possible to use a small sample size to establish associations between physiological or pathological conditions and corresponding changes in urine. ${ }^{14}$ Therefore, screening potential disease biomarkers in animal models followed by validation in human samples may be a good strategy for discovering urinary biomarkers.

Human malaria is a life-threatening disease caused by Plasmodium falciparum and has an estimated annual global disease burden 207 million clinical episodes with 0.6 million deaths in $2012.15,1685 \%$ of the cases and $90 \%$ of the mortality occurs in sub-Saharan Africa, mostly amongst children. ${ }^{17}$ Accurate diagnosis of ill patients has been essential for malaria control programs aimed at reducing morbidity and mortality.

However, the current malaria diagnostic methods are mainly based on blood, in recent years, malaria has been detected by sampling of saliva and urine with mitochondrial PCR-based malaria test Urine Malaria Test. ${ }^{18-20}$ Moreover, urinary biomarkers hold promise for the development of new point-of-care diagnostics for tuberculosis (TB), as 10 mycobacterial proteins were identified exclusively in the urine of definite TB patients and 6 mycobacterial proteins were detected exclusively in the urine of presumed LTBI patients. ${ }^{21}$ Hence, the aim of this investigation was attempted to identify the parasite proteins and the significantly altered mouse proteins in the urine of mice infected by Plasmodium yoelii. 


\section{Materials and methods}

\section{Animals and ethics statement}

Pathogen-free, Female Balb/c mice 6-8 weeks of age were purchased from the Institute of Laboratory Animal Science, Chinese Academy of Medical Science \& Peking Union Medical College (Beijing, China). All animals were fed standard laboratory diet and kept under controlled temperature $\left(22 \pm 1^{\circ} \mathrm{C}\right)$ and humidity $(65-70 \%)$. The study was performed after one week of acclimation. This study was approved by the Institute of Basic Medical Sciences Animal Ethics Committee, Peking Union Medical College (Animal Welfare Assurance Number: A5518). All mice received humane care in compliance with the institutional animal care guidelines approved by the Institutional Animal Care and Use Committee of the Peking Union Medical College.

\section{Experimental infection and urine collection}

Lethal P. yoelii $17 X \mathrm{~L}$ malaria parasites were maintained in Balb/c mice. Infections were initiated by intraperitoneal injection of $2 \times 10^{5}$ $P$. yoelii-infected erythrocytes..$^{22}$ The parasitemia of individual mice was monitored via blood smears stained with Giemsa. 30 mice were randomly divided into 6 groups ( 5 per group), the urine samples were collected before and 1, 2, 3, 4, 5 days after infected using metabolic cages.

\section{Acetone precipitation}

All urine samples were centrifuged at $5000 \mathrm{~g}$ for $30 \mathrm{~min}$, and the pellets were discarded. The supernatants were precipitated with $75 \%$ $\mathrm{v} / \mathrm{v}$ acetone for $12 \mathrm{~h}$ followed by centrifugation at $12000 \mathrm{~g}$ for $30 \mathrm{~min}$. After removing the supernatant, the pellets were thoroughly air-dried, resuspended in lysis buffer ( $8 \mathrm{M}$ urea, $2 \mathrm{M}$ thiourea, $50 \mathrm{mM}$ Tris and $25 \mathrm{mM}$ DTT) and subjected to protein quantitation by the Bradford assay.

\section{ID-SDS PAGE and in-gel digestion}

Urinary proteins were dissolved in PAGE sample buffer $(50 \mathrm{mM}$ Tris- $\mathrm{HCl}, \mathrm{pH} 6.8$, containing $50 \mathrm{mM}$ DTT, $0.5 \%$ SDS, and $10 \%$ glycerol), incubated at $57^{\circ} \mathrm{C}$ for $5 \mathrm{~min}$ and resolved by 1D-SDS PAGE. After electrophoresis, each gel band was cut into slices according to the molecular weight. The gel slices were washed twice by incubation in $100 \mathrm{mM}$ ammonium bicarbonate for $15 \mathrm{~min}$ at $37^{\circ} \mathrm{C}$, followed by $100 \mathrm{mM}$ ammonium bicarbonate/acetonitrile $(1: 1)$ for $15 \mathrm{~min}$ at $37^{\circ} \mathrm{C}$. After cooling to room temperature, the samples were reduced using $20 \mathrm{mM}$ dithiothreitol with heating at $56^{\circ} \mathrm{C}$ for 1 hour, followed by alkylation using $55 \mathrm{mM}$ iodoacetamide at room temperature for 1 hour in the dark. The gel slices were dried, and sequence-grade modified trypsin was added at a protein-to-enzyme ratio of 30:1, followed by incubation overnight at $37^{\circ} \mathrm{C}$. After trypsin digestion, the supernatant was collected. The resulting peptides were extracted from the gel slices by incubating once in $50 \mathrm{mM}$ ammonium bicarbonate for 1 hour at $37^{\circ} \mathrm{C}$ and twice in $5 \%$ formic acid/acetonitrile (1:1) for 1 hour at $37^{\circ} \mathrm{C}$. The three collected extractions were pooled with the initial digestion supernatant, desalted by solid-phase extraction (Oasis column; Waters, Inc., Milford, Massachusetts, USA), dried in a SpeedVac, resuspended with $10 \mu \mathrm{L} 0.1 \%$ formic acid, and stored at $-80^{\circ} \mathrm{C}$ until use (Figure 1).

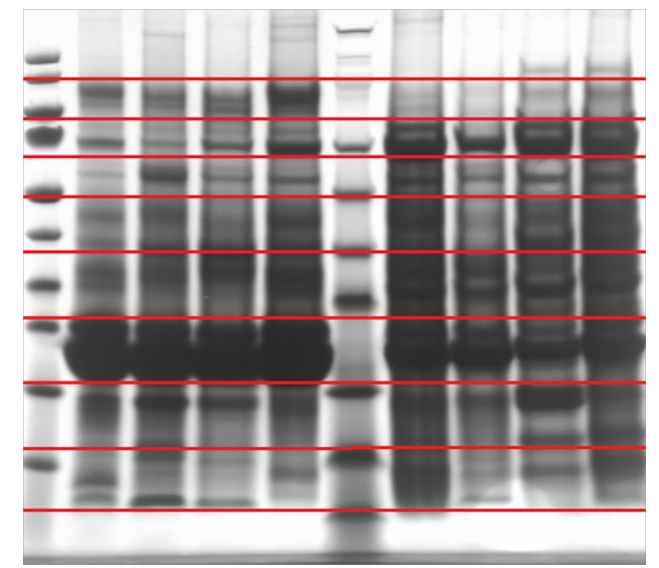

Figure I ID-SDS PAGE and in-gel digestion.

\section{Nano LC-MS/MS analysis}

Lyophilized peptides from the urine samples of 4 days after infected were redissolved in $0.1 \%$ formic acid and subjected to chromatography using a Waters Ultraperformance LC system. Peptides were separated on a 10-cm fused silica column packed in-house using ReproSilPur C18- AQ ( $3 \mu \mathrm{m}$ resin). Elution was performed over a gradient of $5-28 \%$ buffer B $(0.1 \%$ formic acid, $99.9 \% \mathrm{ACN}$; flow rate, $0.3 \mu \mathrm{L} /$ $\mathrm{min}$ ) for $60 \mathrm{~min}$. The remaining peptides were eluted using a short gradient from 28 to $100 \%$ buffer B in 5 min. The eluted peptides were analyzed using a Triple TOF 5600 MS system. The MS data were acquired using an ion spray voltage of $3 \mathrm{kV}$, curtain gas of $20 \mathrm{PSI}$, nebulizer gas of $30 \mathrm{PSI}$, and an interface heater temperature of $150^{\circ} \mathrm{C}$. The precursors were acquired in $500 \mathrm{~ms}$ ranging from 350 to $1250 \mathrm{Da}$, and the product ion scans were acquired in $50 \mathrm{~ms}$ ranging from 250 to $1800 \mathrm{Da}$. A rolling collision energy setting was used. A total of 30 product ion scans were collected if exceeding a threshold of 125 counts per second (counts/s) and with a +2 to +5 charge state for each cycle. Each sample was analyzed once.

The resulting peptides from the urine samples of 5 days after infected were analyzed on Orbitrap Fusion Lumos mass spectrometer (Thermo Electron, Bremen, Germany) interfaced with Easy-nLC 1200 nanoflow liquid chromatography system (Thermo Scientific, Odense, Denmark). Peptide digests were reconstituted in $0.1 \%$ formic acid and loaded ontotrap column packed $(75 \mu \mathrm{m} \times 2 \mathrm{~cm})$ with Magic C18 AQ(Michrom Bioresources, Inc., Auburn, CA) at a flow rate of $3 \mu \mathrm{L} / \mathrm{min}$. Peptides were separated on an analytical column $(75 \mu \mathrm{m} \times 20 \mathrm{~cm})$ at a flow rate of $400 \mathrm{~nL} / \mathrm{min}$ using a step gradient of 5-2 \% solvent B ( $0.1 \%$ formic acid in $95 \%$ acetonitrile) for first $110 \mathrm{~min}$ and $25-40 \%$ solvent B for $110-140 \mathrm{~min}$. The total run time was set to $35 \mathrm{~min}$. Mass spectrometer was operated in data-dependent acquisition mode. A survey full scan MS (from m/z 350-1700) was acquired in the Orbitrap with resolution of 120,000 at $400 \mathrm{~m} / \mathrm{z}$. Most intense fifteen precursor ions with charge state $\geq 2$ were isolated and fragmented using HCD fragmentation with $30 \%$ normalized collision energy and detected at a mass resolution of 30,000 at $400 \mathrm{~m} / \mathrm{z}$. Dynamic exclusion was set for $30 \mathrm{~s}$ with a 10ppm mass window.

\section{Database searching and protein quantification}

Mascot Daemon software (version 2.4.0, Matrix Science, London, 
UK) was used to search the MS/MS data against the SwissProt Plasmodium yoelii, falciparum and vivax database to identify the parasite proteins parasite proteins in urine. For the identification of host (mouse) urinary proteins the raw data were searched against SwissProt mouse database. Carbamidomethylation of cysteines was set as a fixed modification, and oxidation of methionine and protein $\mathrm{N}$-terminal acetylation were set as variable modifications. The specificity of trypsin digestion was set for cleavage after $\mathrm{K}$ or $\mathrm{R}$, and two missed trypsin cleavage sites were allowed. For the data of Triple TOF 5600, the mass tolerances in MS and MS/MS were both set to $0.05 \mathrm{Da}$, and they were set to $10 \mathrm{ppm}$ and $0.06 \mathrm{Da}$ respectively for the data of Orbitrap Fusion Lumos. Peptide identifications were accepted if they were detected with $90.0 \%$ probability and an FDR less than $0.1 \%$ by the Scaffold local FDR algorithm. Protein identifications were accepted if they were detected with an FDR of less than $1 \%$ and contained at least 2 identified peptides. Label-free quantification was performed using the Scaffold software (version Scaffold 4.4.0, Proteome Software Inc., Portland, OR, USA). Proteins that contained similar peptides and could not be differentiated based on MS/MS analysis alone were grouped to satisfy the principles of parsimony (Supplementary Table 1) (Supplementary Table 2).

\section{Results and discussion}

\section{Experimental infection and urine collection}

Some of the mice developed gross hematuria 6 days after infected by $P$. yoelii $17 X L$ malaria parasites, so the urine samples were suspended at the same time. On the seventh day after infection, all the mice suffered from severe parasitemia with more than $70 \%$ of erythrocytes infected by malaria parasites, and early half of the mics were died (Table 1) (Table 2).

Table I Parasitemia of mice monitored via blood smears stained with Giemsa(percent of infected erythrocytes).

\begin{tabular}{lllllll}
\hline & Group I & Group 2 & Group 3 & Group 4 & Group 5 & Group 6 \\
\hline Day 4 & $2.7 \%$ & $3.1 \%$ & $3.5 \%$ & $3.0 \%$ & $3.5 \%$ & $1.9 \%$ \\
Day 5 & $11.6 \%$ & $11.7 \%$ & $11.2 \%$ & $10.5 \%$ & $12.5 \%$ & $10.1 \%$ \\
Day 6 & $47.2 \%$ & $40.2 \%$ & $43.5 \%$ & $47.3 \%$ & $42.5 \%$ & $30.7 \%$ \\
Day 7 & $65.7 \%$ & $75.4 \%$ & $71.0 \%$ & $81.4 \%$ & $75.1 \%$ & $82.0 \%$ \\
\hline
\end{tabular}

Table 2 The number of survived mice and mice with gross hematuria.

\begin{tabular}{|c|c|c|c|c|c|c|c|}
\hline & & Group I & Group 2 & Group 3 & Group 4 & Group 5 & Group 6 \\
\hline Day 4 & survival & 5 & 5 & 5 & 5 & 5 & 5 \\
\hline Day 5 & survival & 5 & 5 & 5 & 5 & 5 & 5 \\
\hline \multirow[t]{2}{*}{ Day 6} & survival & 5 & 5 & 5 & 5 & 5 & 5 \\
\hline & $\begin{array}{l}\text { grossh } \\
\text { ematuria }\end{array}$ & 2 & 2 & 3 & I & I & \\
\hline Day 7 & survival & 2 & 3 & 2 & 3 & 2 & 4 \\
\hline Day 8 & survival & 0 & I & 0 & I & 0 & I \\
\hline
\end{tabular}

Detection of parasite proteins in the urine of mice infected by P. yoelii I7XL malaria parasites

According to the literature, parasitemia will be detected via blood smears stained with Giemsa 3 days after infection. Therefore, urine samples on the fourth and fifth days after infection were used to detect parasite proteins. In order to improve the depth of the identification, the urinary proteins were separated by SDS PAGE and each gel band was cut into 10 slices, respectively, according to the molecular weight. After trypsin digestion, resulting peptides from the urine samples of 4 days after infection were analysed by a Triple TOF 5600 MS system. 427 mouse proteins were identified after database searching; however, there were no reliable parasite proteins detected in the urine, although the MS data were searched against not only the SwissProt Plasmodium yoelii database but also the SwissProt_Plasmodium falciparum and vivax database (the other two common forms).

The reason may be that parasitemia is about $3 \%$ of erythrocytes infected on the fourth day after infection. As a result, urine samples of mice 5 days after infection (approximately 10\% of erythrocytes infected) were analyzed using the Orbitrap Fusion Lumos MS system, the best mass spectrometer at present, under the same strategy. The depth of the protein identification was further improved with 821 mouse proteins identified. However, after searching against the above three plasmodium database, there were still no reliable parasite proteins detected in the urine, even at low MW range, indicating that extrinsic protein may be mostly degraded in blood.

\section{Identification of the significantly changed host (mouse) proteins in urine}

There were 427 and 821 mouse proteins identified in the urine of 4 and 5 days after infection, respectively. Under the criteria $p<0.05$, fold change $>2$ and the spectral count $>5,21$ and 83 proteins were significantly altered after quantified by Scaffold. There are only 4 differential proteins (Mucin-2, Galectin-3-binding protein, Ig alpha chain $C$ region, Apolipoprotein D) shared in the urine on both the 4 th and 5th days after infection and all of them are significantly increased after infection. 8 proteins of the 21 proteins (about one third) altered on the 4 th after infection are related to host defense or immune response, and only one protein (afamin) is reported to be biomarker for renal disease, ${ }^{23}$ suggesting that most differential proteins may be 
associated with malaria. However, on the 5th after infection, 26 of the 83 proteins (especially 20 of the 40 high abundant proteins) are biomarkers for renal diseases, ${ }^{8}$ indicating that the kidney are seriously injured, which may be the reason why there are only 4 proteins shared between these two days.

According to the functional annotations of the Uniprot database, Ig alpha is the major immunoglobulin class in body secretions, which may serve to defend against local infection; Galectin-3-binding protein may stimulate host defense against viruses and tumor cells; Mucin- 2 coats the epithelia of the intestines, airways, and other mucus membrane-containing organs, providing a protective, lubricating barrier against particles and infectious agents at mucosal surfaces. Apolipoprotein D was proven to have a role in preventing lipid peroxidation, especially in the central nervous system, protecting the cells from oxidative stress caused by infection. ${ }^{24}$

\section{Conclusion}

In conclusion, these 4 proteins may be candidate urinary biomarkers for malaria; however, as it is the first attempt to search urinary biomarkers for infectious diseases to our knowledge, the specificity need to be explored in further studies on other infectious diseases.

\section{Acknowledgements}

This work was supported by National Key Research and Development Program of China (2016 YFC 1306300), Key Basic Research Program of the Ministry of Science and Technology of China (2013FY114100), Beijing Natural Science Foundation (7173264, 7172076), Beijing cooperative construction project (110651103) Beijing Normal University (11100704) Peking Union Medical College Hospital (2016-2.27).

\section{Conflict of interest}

The authors declare no competing interests.

\section{References}

1. Wu J, Zhao HR, Zhang HY, et al. Thrombin generation increasing with age and decreasing with use of heparin indicated by calibrated automated thrombogram conducted in Chinese. Biomed Environ Sci. 2014;27(5):378-384.

2. Gao Y. Urien-an untapped goldmine for biomarker discovery? $S c i$ China Life Sci. 2013;56(12):1145-1146.

3. Gao Y. Differences in Blood and Urine Biomarker Discovery. MOJ Proteomics \& Bioinformatics. 2015;2(5):00058.

4. Li M, Zhao M, Gao Y. Changes of proteins induced by anticoagulants can be more sensitively detected in urine than in plasma. Sci China Life Sci. 2014;57(7):649-656.

5. Huang JT, Chaudhuri R, Albarbarawi O, et al. Clinical validity of plasma and urinary desmosine as biomarkers for chronic obstructive pulmonary disease. Thorax. 2012;67(6):502-508.

6. Wu T, Du Y, Han J, et al. Urinary angiostatin - a novel putative marker of renal pathology chronicity in lupus nephritis. Mol Cell Proteomics 2013;12(5):1170-1179.
7. Gao Y (2014) Opportunities you do not want to miss and risks you cannot afford to take in urine biomarker era. MOJ Proteomics Bioinformatics 1(1): 00003

8. Shao C, Li M, Li X, et al. A tool for biomarker discovery in the urinary proteome: a manually curated human and animal urine protein biomarker database. Mol Cell Proteomics. 2011;10(11):M111 010975.

9. Guo Z, Zhang Y, Zou L, et al. A Proteomic Analysis of Individual and Gender Variations in Normal Human Urine and Cerebrospinal Fluid Using iTRAQ Quantification. PLoS One. 2015;10(7):e0133270.

10. Zhao M, Li X, Li M. Effects of anesthetics pentobarbital sodium and chloral hydrate on urine proteome. Peer J. 2015;3:e813.

11. Li X, Zhao M, Li M. Effects of three commonly-used diuretics on the urinary proteome. Genomics Proteomics Bioinformatics. 2014;12(3):120-126.

12. Kohler M, Franz S, Regeniter A, et al. Comparison of the urinary protein patterns of athletes by 2D-gel electrophoresis and mass spectrometry-a pilot study. Drug Test Ana. 2009;1(8):382-386.

13. Airoldi L, Magagnotti C, Iannuzzi AR, et al. Effects of cigarette smoking on the human urinary proteome. Biochem Biophys Res Commun. 2009;381(3):397-402.

14. Gao Y. Roadmap to the Urine Biomarker Era. MOJ Proteomics Bioinform. 2014;1:00005.

15. Snow RW, Guerra CA, Noor AM, et al. The global distribution of clinical episodes of Plasmodium falciparum malaria. Nature. $2005 ; 434(7030): 214-217$.

16. Hay SI, Okiro EA, Gething PW, et al. Estimating the global clinical burden of Plasmodium falciparum malaria in 2007. PLoS Med. 2010;7(6):e1000290.

17. Bryce J, Boschi-Pinto C, Shibuya K, et al. WHO estimates of the causes of death in children. Lancet. 2005;365(9465):1147-1152.

18. Ghayour Najafabadi Z, Oormazdi H, Akhlaghi L, et al. Mitochondrial PCR-based malaria detection in saliva and urine of symptomatic patients. Trans R Soc Trop Med Hyg. 2014;108(6):358-362.

19. Oguonu T, Shu E, Ezeonwu BU, et al. The performance evaluation of a urine malaria test (UMT) kit for the diagnosis of malaria in individuals with fever in south-east Nigeria: cross-sectional analytical study. Malar J. 2014; 13:403.

20. Oyibo WA, Ezeigwe N, Ntadom G, et al. Multicenter Pivotal Clinical Trial of Urine Malaria Test for Rapid Diagnosis of Plasmodium falciparum Malaria. J Clin Microbiol. 2016;55(1):253-263.

21. Young BL, Mlamla Z, Gqamana PP, et al. The identification of tuberculosis biomarkers in human urine samples. Eur Respir J. 2014;43(6):1719-1729.

22. Shao D, Zhong X, Zhou YF, et al. Structural and functional comparison of MIF ortholog from Plasmodium yoelii with MIF from its rodent host. Mol Immunol. 2010;47(4):726-737.

23. Rao PV, Lu X, Standley M, et al. Proteomic identification of urinary biomarkers of diabetic nephropathy. Diabetes Care. 2007;30(3):629 637.

24. Ganfornina MD, Do Carmo S, Lora JM, et al. Apolipoprotein D is involved in the mechanisms regulating protection from oxidative stress. Aging Cell. 2008;7(4):506-515. 